\title{
On QoS Constraints and Joint Source-Channel Coding for Real-Time Cooperative Multimedia Communications
}

\author{
Andres Kwasinski, H. Vicky Zhao, and K. J. Ray Liu \\ Department of Electrical and Computer Engineering, University of Maryland, USA. \\ $\{$ ak,hzhao,kjrliu\}@umd.edu
}

\begin{abstract}
Cooperative diversity exploits the broadcast nature of wireless channels by allowing users to relay information for each other so as to create multiple signal paths. This technique could be used together with joint source-channel bit rate allocation to transmit conversational multimedia traffic. This paper studies, following a methodology that avoids the use of high or low SNR approximations, the interaction between joint source-channel bit rate allocation with $\mathrm{AF}$ and DF cooperation. Furthermore, the effect of QoS constraints on performance is studied. In addition to the advantages of user-cooperation at medium and low source-destination channel Signal-to-Noise ratio, the results in this paper point at the fact that significant extra performance gains could be obtained by careful selection of the relay node. Guidelines for choosing the relay are also discussed.
\end{abstract}

\section{INTRODUCTION}

Future wireless communications will need to support conversational multimedia traffic with good quality of service while operating at reduced power levels in environments impaired by signal fading. One effective way in overcoming this challenge is the use of diversity techniques. In spatial diversity, copies of a signal are transmitted through paths ideally undergoing mutually independent fading by using multiple physically-separated antennas at the transmitter, the receiver or both. Unfortunately, practical implementation of spatial diversity is limited by the size of mobile terminals. This limitation can be overcome by making users collaborate during communication and relay information for each other so as to create multiple signal paths that are combined at the receiver, a technique known as cooperation diversity [1]. Cooperation diversity builds upon early studies on the relay channel [2]. While [1] introduced the idea of cooperation through "decode and forward" (DF), [3] introduced the idea of implementing cooperation through "amplify and forward" (AF) and studied the achievable capacity of user-cooperation schemes. Diversity through coded cooperation was studied in [4].

Joint source-channel bit rate allocation is a technique by which the available bit rate is allocated between source encoding bit rate and channel coding rate based on a performance measure that jointly considers the effects of source and channel coding. This technique is useful in allocating resources for conversational traffic that uses end-to-end distortion as performance measure and which cannot resort to ARQ techniques due to strict delay constraints. User cooperation diversity can be combined with joint source-channel coding to transmit multimedia sources. Recently, there has been a limited number of works studying this problem. In [5], the authors considered a capacity achieving case and used high SNR based analysis. AF cooperation was combined with refinable single description coding with only the base layer transmitted using cooperation. In [6], this approach was used to study layered encoding with coded cooperation, and the work in [7] studied transmission of multi layered sources using AF cooperation and a broadcast strategy that superimpose the layers during transmission. In [8], we studied how to combine source coding diversity (through multiple description coding) with $\mathrm{AF}$ and DF cooperation. In [9], we presented the concept of multimedia cooperative communication, and compared single description, multiple description and layered coding combined with $\mathrm{AF}, \mathrm{DF}$ and coded cooperation. The general approach is to study the joint source-channel bit rate allocations that a conversational multimedia transmission protocol should make before transmission in each period. These works showed that cooperation provides useful gains in all cases where the direct channel between the source and the destination is not good. Also, we noticed that in most cases DF cooperation yields the best performance while coded cooperation suffers from inefficiency when feedback is not present.

In this paper, we study the interaction between joint sourcechannel bit rate allocation with $\mathrm{AF}$ and $\mathrm{DF}$ cooperation. We do so by avoiding the use of high SNR approximations because they may hold for only a few of the channel codes used in the optimization. For DF cooperation we consider a setup tailored to the transmission of conversational multimedia that avoids relay idling after a decoding failure. In this setup cooperatives nodes are organized in pairs with each member performing both as source and relay node. Our analysis shows that while AF cooperation behaves similarly to direct transmission, DF behaves very differently. We observe that the use of joint source-channel bit rate allocation with the considered DF cooperation scheme yields the best performance when the source-relay channel SNR is either high or low but not inbetween. In addition, we study how QoS constraints affect the performance of joint source-channel bit rate allocation when combined with AF or DF cooperation. In this case we consider FER constraints, which are typical of conversational multimedia communications. Our conclusion here reinforces the observation that the best performance is obtained by 
carefully choosing a relay node that ensures operation at either high or low source-relay channel SNR.

\section{SYSTEM MODEL}

We consider a wireless network shared between users by allocating to each call an orthogonal channel with fixed communication capacity $F$ channel code symbols per transmission period. We focus on a source node transmitting conversational multimedia traffic to a destination node. At the source node, a block of $N$ input signal samples (each modeled as a memoryless, zero-mean, unit-variance Gaussian source) are first compressed at a source encoder and then error-protected for transmission over a fading channel that remains constant during each transmission period. Our study takes the viewpoint of a resource allocation protocol that decides what the best decision is to transmit the conversational multimedia source given the channel states during a transmission period.

We assume that the source signal samples are first fed into a source encoder that generates one coded representation of the source samples (a single bit stream) at a rate $R_{S}$ bits per source sample. The performance of source codecs can be measured through its achievable distortion rate (D-R) function. The D-R function for SD source codecs is frequently considered to be of the form $D_{S}\left(R_{S}\right)=c_{1} 2^{-c_{2} R_{S}}$. This form of D-R function can approximate or bound a wide range of practical systems such as video coding with an MPEG codec [10], speech using a CELP-type codec [11], or when the high rate approximation holds. Assuming that each of the input signal samples are memoryless, following a zero-mean, unit-variance Gaussian distribution and if long block source codes are used, we have $c_{1}=1, c_{2}=2$ ( [12]). Thus, without loss of generality, we assume,

$$
D_{S}\left(R_{S}\right)=2^{-2 R_{S}}
$$

After source encoding, the source-encoded bits are protected against transmission errors through a channel code. We assume that the delay constraint excludes the use of capacity-achieving codes or ARQ-based error control. We consider Reed-Solomon block codes operating on $b$ bits symbols with parameters $(n, k)$, i.e. each operates at a rate $r=k / n$, encoding $k$ symbols into an $n$-symbols codeword. Since in our previous works [8], [9], we noticed little dependence of the optimal performance on the choice for $n$, we choose to keep this value fixed. Also, we assume that the receiver discards those blocks of data that have remaining errors after channel decoding. This is common practice in conversational communications due to the strict delay constraints. If a channel frame contains $L$ codewords, the probability of having a frame with errors is $\widetilde{P}(\gamma, L)=1-(1-q(\gamma))^{L}$, where $\gamma$ is the channel signal to noise ratio (SNR) and $q(\gamma)$ is the probability of channel decoder failure when using a bounded distance decoder [13]. For the case of Reed-Solomon codes we have this probability

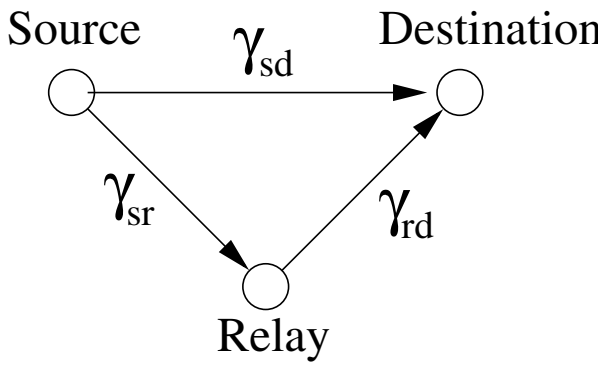

Fig. 1. Simplified wireless network with user cooperation.

approximated as

$$
\begin{aligned}
q(\gamma) & =P\left[\text { erred symbols in codeword }>\left\lfloor\frac{n-k}{2}\right\rfloor\right] \\
& =\sum_{j=t}^{n}\left(\begin{array}{l}
n \\
j
\end{array}\right) P_{s}(\gamma)^{j}\left(1-P_{s}(\gamma)\right)^{n-j}
\end{aligned}
$$

where $t=1+\left\lfloor\frac{n-k}{2}\right\rfloor$ and $P_{s}$ is the probability of a symbol error. For $b$-bits symbols, $P_{s}(\gamma)=1-\left(1-P_{b}(\gamma)\right)^{b}$, where $P_{b}$ is the bit error probability and depends on the modulation scheme as well as the channel conditions. In this work, we will assume BPSK modulation over AWGN channel with coherence detection and maximum-likelihood decoding.

Communication between a source and a destination node may be carried on using or not user cooperation. In a cooperative scheme a third node, the relay node, is associated with the source node to achieve user-cooperation diversity (see Figure 1). Communication in a cooperative setup takes place in two phases, which share the fixed communication capacity $F$ due to our requirements for orthogonal channels. In phase 1 , a source node sends information to its destination node. Due to the broadcast nature of wireless communications, the source transmission is also overheard by the relay node. In phase 2 , the relay cooperates by forwarding to the destination the overheard information. At the receiver, the signals received from the source and the relay are combined to detect the transmitted message. We assume that a Maximum Ratio Combiner (MRC) is used to combine the symbols arriving through different paths. Note that in order to implement the two-phase operation, the total bandwidth allocated to a call needs to be split. We assume that the bandwidth is evenly split between the two phases. In our setup, we will also assume a symmetric setting with reciprocal source-relay channels and where the source cooperates with the relay and vice versa (during phase 2 the source relays the information sent by the relay). Depending on the different processing done at the relay, there are several different strategies to implement user-cooperation. We will consider two schemes: amplify-and-forward (AF) and decodeand-forward (DF).

In amplify-and-forward, the relay retransmits the source's signal without further processing other than power amplification. It was shown in [3] that for symbols transmitted using AF cooperation, the SNR at the receiver after the MRC is

$$
\gamma_{A}=\gamma_{s d}+\frac{\gamma_{s r} \gamma_{r d}}{1+\gamma_{s r}+\gamma_{r d}}
$$

where $\gamma_{s d}$ is the source-destination channel SNR, $\gamma_{s r}$ is the source-relay channel SNR and $\gamma_{r d}$ is the relay-destination 
channel SNR. The probability of having a frame with errors will be

$$
\widetilde{P_{A}}\left(\gamma_{A}, L\right)=1-\left(1-q\left(\gamma_{A}\right)\right)^{L},
$$

with $q(\cdot)$ as in (2).

In decode-and-forward, the relay first decodes the message from the source. If the decoded message has no errors, the relay re-encodes it and transmits a copy. In a possible DF scheme, if the relay fails to decode the message, it idles until the next is received. The problem with this approach is that it wastes valuable bandwidth for the transmission of realtime multimedia. To address this drawback, we consider that all nodes that may cooperate are active in a call. With this assumption, nodes may be paired up in such a way that two active nodes help each other. In this scheme when one of the two nodes fails decoding its partner's message, it sends during phase 2 a copy of its own signal. Because of the inherent symmetry of this setup we will focus on the performance of one of the two partners, which we will still call the source node while its partner will be named the relay node. It can be shown, [3], that for those symbols transmitted using DF cooperation, the SNR at the receiver after the MRC is $\gamma_{D}=2 \gamma_{s d}$ if source and relay fail decoding, $\gamma_{D}=\gamma_{s d}+\gamma_{r d}$ if source and relay succeed, $\gamma_{D}=\gamma_{s d}$ if source succeeds and relay fails and $\gamma_{D}=2 \gamma_{s d}+\gamma_{r d}$ if source fails and relay succeeds. The probability of having a frame with errors is

$$
\begin{aligned}
\widetilde{P}_{D}\left(\gamma_{D}, L\right)= & \widetilde{P}_{s f r f} \widetilde{P}_{s r}^{2}+\widetilde{P}_{s s r s}\left(1-\widetilde{P}_{s r}\right)^{2}+ \\
& +\left[\widetilde{P}_{s s r f}+\widetilde{P}_{s f r s}\right] \widetilde{P}_{s r}\left(1-\widetilde{P}_{s r}\right),
\end{aligned}
$$

where $\widetilde{P}_{s r}, \widetilde{P}_{s f r f}, \widetilde{P}_{s s r s}, \widetilde{P}_{s s r f}$ and $\widetilde{P}_{s f r s}$ are computed using (2) with $\gamma=\gamma_{s r}, \gamma=2 \gamma_{s d}, \gamma=\gamma_{s d}+\gamma_{r d}, \gamma=\gamma_{s d}$, and $\gamma=2 \gamma_{s d}+\gamma_{r d}$, respectively, and an $(n, k)$ code [9].

\section{QoS Constrained Joint Source-Channel CODING AND COOPERATIVE COMMUNICATION}

In this section, we study the use of QoS constrained joint source-channel coding to send conversational multimedia traffic using a cooperative scheme.

Constraints that involve QoS considerations are essential to any communications system design. In problems such as the ones considered in this paper where end-to-end distortion is the performance measure being optimized, it is important to also impose constraints on distortion-related parameters. When considering conversational traffic, it is important to constrain the error rate during transmission because the strict delay constraint prevents the use of ARQ schemes. Error rate is typically constrained through the frame error rate (FER) or the bit error rate (BER). In this paper, we focus on FER as the QoS constraint. The actual value for this constraint is highly dependant on the system setup, such as type of source, source codec used, performance of the error concealment scheme being used, etc. We will denote by $Q$ the particular QoS constraint (FER in this paper). In the case of speech, a typical value for $Q$ is $10^{-2}$ [14]. Other sources, such as video, require lower values for $Q$ in order to deliver good quality.
In the absence of user-cooperation, the problem of joint source-channel bit rate allocation consists of allocating source coding rate and channel coding rate with the goal of minimizing average end-to-end distortion. If each of the $N$ source samples is source encoded using $R_{S N}$ bits and error protected with a $(n, k)$ code, there are $L=N R_{S N} /(b k)$ codewords per frame. With $F=L n$, we have $R_{S N}=b k F /(n N)$ and the problem of joint source-channel bit rate allocation can be formulated as

$$
\begin{array}{ll}
\min _{k} & \left\{D_{F} \widetilde{P}\left(\gamma_{s d}, L\right)+D_{S}\left(R_{S N}\right)\left(1-\widetilde{P}\left(\gamma_{s d}, L\right)\right)\right\} \\
\text { s.t. } & \widetilde{P}\left(\gamma_{s d}, L\right)<Q .
\end{array}
$$

where $D_{F}$ is the distortion when the frame is received with errors $\left(D_{F}=1\right.$ for our source model), $\gamma_{s d}$ is the source-destination channel SNR and the constraint takes into consideration the QoS restrictions. Note that because the communication capacity allocated to a call $(F)$ and the number of symbols in a channel codeword $(n)$ are both assumed fixed, choosing the optimal channel code determines also the optimum source coding rate.

When using AF cooperation the nature of the problem remains the same. This is because the resulting SNR when using AF cooperation is a translated version of the sourcedestination channel SNR (3). Nevertheless, when using cooperation, the useful communication capacity available for the call gets halved so as to allow for the two-phase cooperation scheme. Therefore, if we use $R_{S C}$ to denote the source coding rate when using cooperation and if the channel code is $(n, k)$, there are $L_{S C}=N R_{S C} /(b k)$ codewords in one frame. These codewords are mapped into $F / 2=n L_{s c}$ channel code symbols. Then, $R_{S C}=b k F /(2 n N)$ and the problem of joint source-channel bit rate allocation can be formulated as

$$
\begin{array}{ll}
\min _{k} & \left\{D_{F} \widetilde{P_{A}}\left(\gamma_{A}, L_{s c}\right)+D_{S}\left(R_{S C}\right)\left(1-\widetilde{P_{A}}\left(\gamma_{A}, L_{s c}\right)\right)\right\} \\
\text { s.t. } & \widetilde{P_{A}}\left(\gamma_{A}, L_{s c}\right)<Q .
\end{array}
$$

Both AF and DF cooperation use the call communication capacity in the same way. Thus, the problem of joint sourcechannel bit rate allocation for DF cooperative communication can be formulated as

$$
\begin{array}{ll}
\min _{k} & \left\{D_{F} \widetilde{P_{D}}\left(\gamma_{D}, L_{s c}\right)+D_{S}\left(R_{S C}\right)\left(1-\widetilde{P_{D}}\left(\gamma_{D}, L_{s c}\right)\right)\right\} \\
\text { s.t. } & \widetilde{P_{D}}\left(\gamma_{D}, L_{s c}\right)<Q .
\end{array}
$$

The important difference between problems (7) and (8) is that when using DF cooperation, the probability of having a frame with errors follows equation (5), which does not involve simple modifications of the source-destination SNR as was the case for AF cooperation. We shall see in section IV, that this difference makes the performance behavior of DF cooperation be markedly different from the one for AF cooperation. 


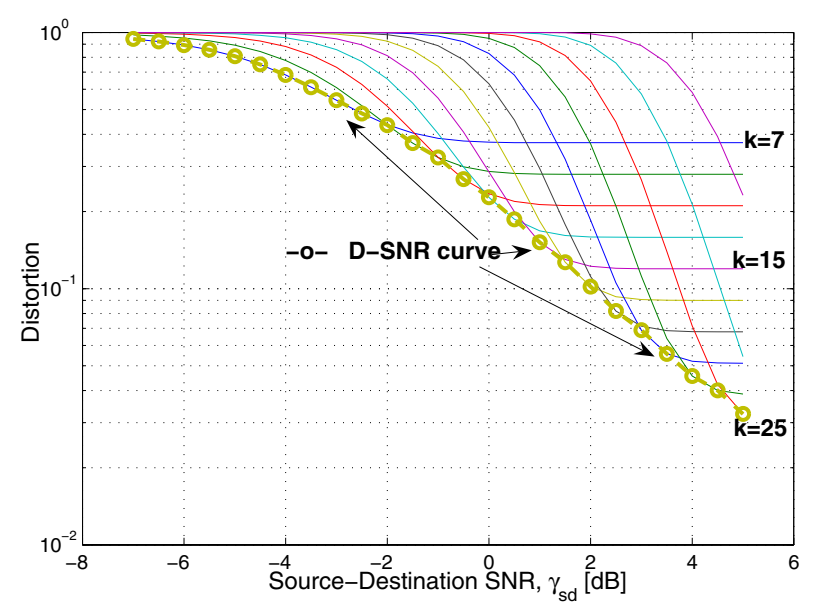

Fig. 2. Joint source-channel allocation with AF cooperation.

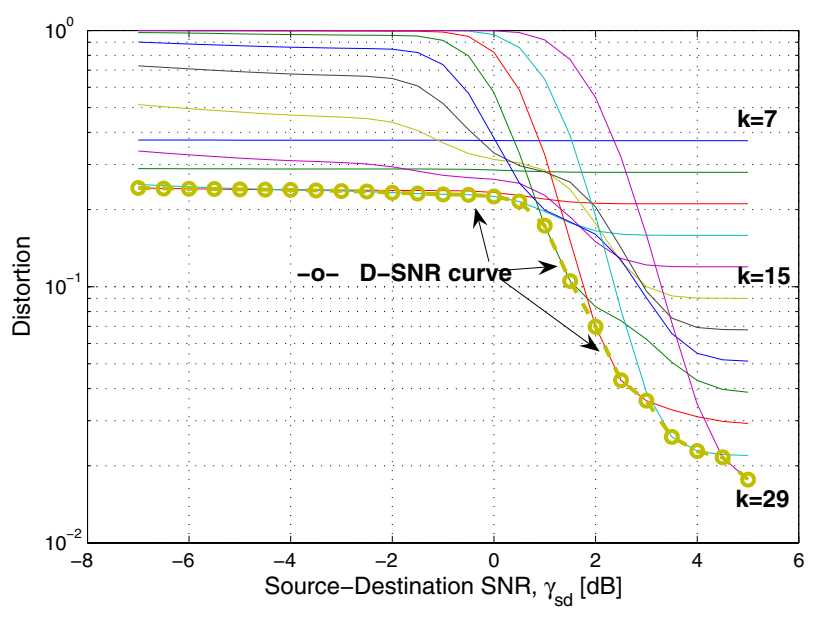

Fig. 3. Joint source-channel allocation with DF cooperation.

\section{Results And AnAlysis}

This section studies the impact of joint source-channel coding and QoS constraints on the design of real time cooperative communication. Our ultimate goal is to investigate the decisions that a protocol should make before transmission of each conversational multimedia frame, including the sourcechannel bit rate allocation, the type of cooperation scheme, etc. We assume that decisions are made before each transmission period based on current channel states. In our work, we consider the source-destination SNR as a variable. Both the source-relay and relay-destination SNRs are parameters that may be thought of as representing the relative position of the relay with respect to the source and the destination. The solution to problems (6) through (8), with or without QoS constraints, is translated into a (minimum) distortion curve as a function of the source destination channel SNR, which we call the D-SNR curve. In all cases, we set $b=5$ bits, $n=31$ channel code symbols, $N=150$ samples and $F=190$ channel code symbols per transmission period and call.

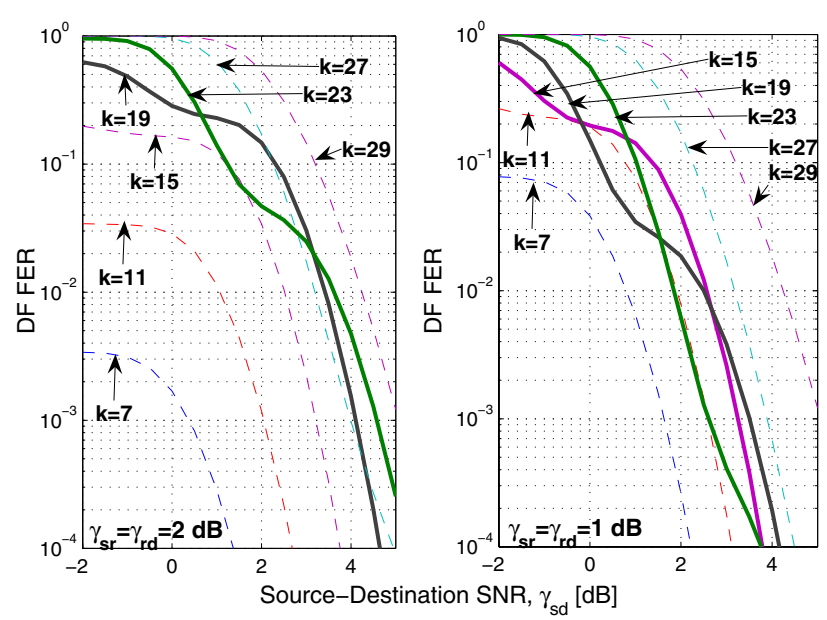

Fig. 4. Frame Error Rate for joint source-channel allocation with DF cooperation. (Left): $\gamma_{s r}=\gamma_{r d}=2 d B$. (Right): $\gamma_{s r}=\gamma_{r d}=1 d B$.

We first study the use of joint source-channel bit rate allocation without QoS constraints. For AF cooperative communication, Figure 2 illustrates the mechanism involved in solving problem (7) when $\gamma_{s r}=\gamma_{r d}=2 d B$. The figure shows the distortion curves for each channel code (equivalently source encoding rate) choice. Also shown, is the D-SNR curve, which yields the minimum distortion by changing the choice of channel code accordingly. This figures reinforces our statement that there is little conceptual difference between the solutions to problems (6) and (7).

For DF cooperation, Figure 3 illustrates the mechanism involved in solving problem (8) with the same setup as Figure 2. The figure shows a markedly different behavior of the distortion curves for each channel code choice. With different channel codes, some distortion curves are smooth, as in Figure 2, while others experience "bumpy" performance. This difference is due to the fact that the frame error probability for each channel code choice now follows Eq. (5). Figure 4 plots the frame error probability for each channel code selection when using DF cooperation. Not surprisingly, the FER shows a behavior similar to the distortion curves. Importantly, Figure 4 shows that stronger codes do not necessarily yield better performance than weaker codes at some channel SNRs. For example, when $\gamma_{s d}=\gamma_{s r}=\gamma_{r d}=1 d B$, the $k=11$ code has worse performance than the $k=19$ code. From Eq. (5), we can see that the three terms in the right-hand side depend on $\widetilde{P}_{s r}$, the decoding failure probability on the source-relay or relay-source link. Therefore, the particular behavior of the FER is due to the fact that, for some range of channel SNR, some channel codes behaves as in the low SNR regime (with the $\widetilde{P}_{s r}^{2}$ term dominating), some channel codes behave as in the high SNR regime (with the $\left(1-\widetilde{P}_{s r}\right)^{2}$ term dominating), and the rest behaves as in neither of the two extremes. It is for these last cases, where no term of (5) dominates, that the "bumpyness" in FER occurs. Note that, in general, high SNR regime would be associated to the strongest channel codes, 


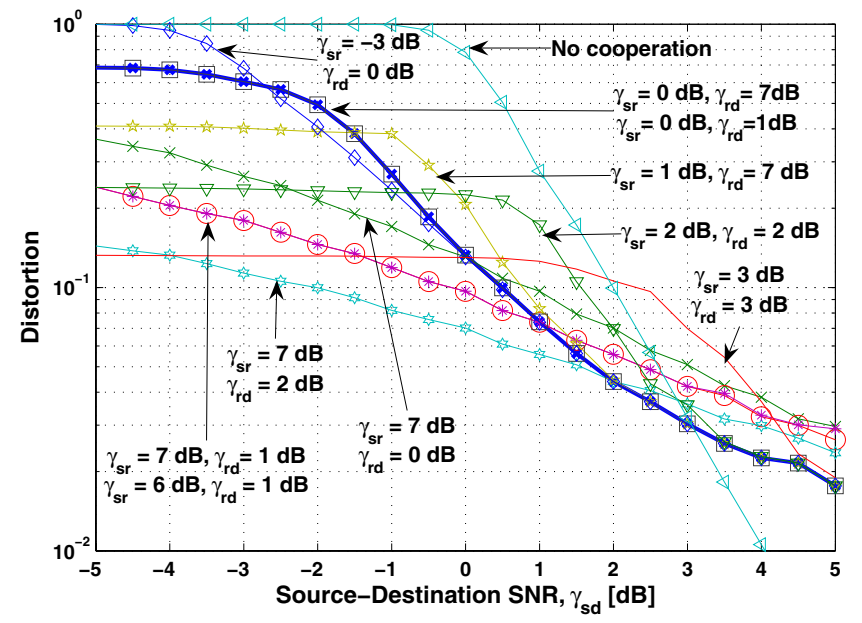

Fig. 5. D-SNR curves for several source-relay-destination channel configurations when using joint source-channel allocation with DF cooperation.

low SNR regime to the weakest and undefined regime to the rest. Yet, as seen in Fig. 4, this association changes with the channel conditions, especially with $\gamma_{s r}$.

Figure 5 shows, for several source-relay-destination channel configurations, the end-to-end distortion of a system that uses joint source-channel allocation with DF cooperation. As is to expect, better source-relay-destination channels yield better performance for low source-destination channel SNR. Surprisingly, for those intermediate values of $\gamma_{s r}$ where no term in (5) dominates, the opposite occurs in the region of intermediate source-destination channel SNR. Part of the reason for this behavior appears in Fig. 3, where we can see that the net effect of the "bumpyness" in distortion is an SNR loss in the D-SNR curve. Figure 4 completes this observation by showing that the set of codes for which "bumpyness" occurs change with $\gamma_{s r}$. As $\gamma_{s r}$ is reduced, the codes affected are increasingly those that yield stronger error protection, and "bumpyness" in FER occurring at smaller values of FER. Therefore, the particular behavior in FER produces a loss in the D-SNR curve for all $\gamma_{s r}$. But at low $\gamma_{s r}$, the variations in FER are relatively (with respect to the value of end-to-end distortion) less intense, and thus yield a much smaller (if not unperceptive) D-SNR loss. This observation should not be taken as a claim of DF cooperation being a bad choice for user-cooperation. In fact, we have observed the contrary in our previous works [8], [9]. This observation points out to the fact that performance could be improved by addressing in the design the inefficiencies implied in our observation. Even more, Figure 5 shows good performance behavior when $\gamma_{s r}$ is either high or low. The operation at high $\gamma_{s r}$ (i.e. a choice of relay close to the source) show the best performance in most of the range of operating states for which cooperation is better than no cooperation. Note that the case of low $\gamma_{s r}$ yields the best performance for a small range of $\gamma_{s d}$. This case does not truly corresponds to a case of cooperative communication because at low $\gamma_{s r}$, both the source and relay fail frequently in decoding each other's messages, and the source switches to non-cooperative operation with $\gamma=2 \gamma_{s d}$. With high $\gamma_{s r}$, both the source and the relay decode each other's messages correctly with high probability, and the corresponding SNR at the receiver side is $\gamma=\gamma_{s d}+\gamma_{r d}$. Therefore, high $\gamma_{s r}$ has better performance than low $\gamma_{s r}$ when $\gamma_{s d}<\gamma_{r d}$, as is confirmed in Figure 5. We finally highlight that this observation, by its own nature, cannot be observed when following high or low SNR approximations.

Figure 6 studies the effects of adding QoS constraints with FER constraint $Q=10^{-3}$. We tested several other setups and we observed that, although qualitative and conceptual results do not change, they become more distinct as the FER constraint is reduced. Overall, the results shown are representative of all the ones we obtained. In Fig. 6 we can first see that there is a range of values of SNR $\gamma_{s d}$ for which the results with FER constraint differs from the ones without. At typical high SNR, the results coincide because any allocation meets the FER constraint. At typical low SNR, the results coincide because no allocation meets the FER constraint and so the minimum distortion solution is chosen. When the results with and without FER constraint differ, the one that considers the constraint always shows larger distortion. This is due to the fact that in order to meet the QoS constraint, a stronger channel code is chosen, which forces the use of a smaller source encoding rate. Nevertheless, the use of this QoS constraint is intended to ensure better subjective quality [14]. For those intermediate values of $\gamma_{s r}$ where no term in (5) dominates $\left(\gamma_{s r}=2 d B\right)$, we can see that DF cooperation is the scheme with performance most affected by the addition of the constraint. As can be seen in Figure 6, this is because the "bumpyness" observed in Figures 4 and 5 makes that some of the points of the D-SNR curve have associated markedly high FER. Then, in order to meet the FER constraint, it becomes necessary to choose a channel code with much stronger error protection to avoid being affected by "bumpyness". The net result of this effect is that with intermediate values of $\gamma_{s r}$, it is better not to use cooperation for a larger range of values of $\gamma_{s d}$. Nevertheless, when the relay is close to or far away from the source (high $\gamma_{s r}$ or low $\gamma_{s r}$, respectively), the performance of DF is once again the best among cooperative schemes and the range of $\gamma_{s r}$ for which cooperation is better does not markedly change. Also, Figure 6 shows that high $\gamma_{s r}$ operation remains the best choice when $\gamma_{s d}<\gamma_{s r}$.

\section{Conclusions}

In this paper, we have studied the interaction between joint source-channel bit rate allocation with AF and DF cooperation. Our methodology avoids the use of high or low SNR approximations, and shows that while AF cooperation behaves similarly to direct transmission, DF behaves very differently. We observed that the particular interaction between joint sourcechannel bit rate allocation and DF cooperation shows that a useful improvement in D-SNR performance could be obtained by operating at either high or low $\gamma_{s r}$. The particular behavior of DF is due to the mathematical structure of the expression for frame error probability. In this paper, we have also studied how much QoS constraints affect the performance of joint sourcechannel bit rate allocation when combined with AF or DF 
cooperation. Considering typical conversational multimedia scenario which prohibits ARQ techniques, we use limits on FER as QoS constraints so as to ensure better subjective quality. Compared with AF cooperation, the QoS constraints impact the performance of DF cooperation by a larger amount when $\gamma_{s r}$ takes intermediate values. The overall conclusion of our study is that performance of cooperative multimedia communication schemes could be greatly improved by careful selection of the relay node, with a focus on the case of DF cooperation (because it shows better performance in most cases). When the SNR values are thought of as representing the relative distance between nodes, we concluded that for the DF scheme we considered, the relay node should not be chosen in an intermediate position between the source and destination.

\section{REFERENCES}

[1] A. Sendonaris, E. Erkip, and B. Aazhang, "User cooperation diversity part I and part II," IEEE Trans. Comm., vol. 51, no. 11, pp. 1927-1948, Nov. 2003.

[2] T. M. Cover and A. A. El Gamal, "Capacity theorems for the relay channel," IEEE Trans. Info. Theory, vol. 25, no. 9, pp. 572-584, September 1979.

[3] J.N. Laneman, D.N.C. Tse, and G.W. Wornell, "Cooperative diversity in wireless networks: Efficient protocols and outage behavior," IEEE Trans. Info. Theory, vol. 50, no. 12, pp. 3062-3080, December 2004.

[4] T. E. Hunter and A. Nosratinia, "Performance analysis of coded cooperation diversity," 2003 Int. Conf. on Comm. ICCO3, vol. 4, pp. 2688-2692, 2003.

[5] D. Gunduz and E. Erkip, "Joint source-channel cooperation: Diversity versus spectral efficiency," in 2004 IEEE Int. Symp. Inf. Theory, JuneJuly 2004, p. 392.

[6] X. Xu, D. Gunduz, E. Erkip, and Y. Wang, "Layered cooperative source and channel coding," in 2005 ICC Multimedia Communication and Home Networking Symposium.

[7] D. Gunduz and E. Erkip, "Source and channel coding for cooperative relaying," in 2005 IEEE Workshop on Sig. Proc. Adv. in Wireless Comm., SPAWC.

[8] A. Kwasinski, Z. Han, and K. J. R. Liu, "Joint source coding and cooperation diversity for multimedia communications," 2005 IEEE Workshop on Sig. Proc. Adv. in Wireless Comm., SPAWC, pp. 129-133.

[9] A. Kwasinski, Z. Han, and K. J. R. Liu, "Cooperative multimedia communications: Joint source coding and collaboration," in Global Telecommunications Conference, GLOBECOM '05, 2005.

[10] Z. He, J. Cai, and C. W. Chen, "Joint source channel rate-distortion analysis for adaptive mode selection and rate control in wireless video coding," IEEE Trans. on Circuits and Systems for Video Technology, vol. 12, no. 6, pp. 511-523, June 2002.

[11] A. Kwasinski, Z. Han, K. J. R. Liu, and N. Farvardin, "Power minimization under real-time source distortion constraints in wireless networks," in IEEE Wireless Communications and Networking Conference (WCNC), New Orleans, Lousiana, March 2003, vol. 1, pp. 532-536.

[12] T. Cover and J. Thomas, Elements of Information Theory, John Wiley Inc., 1991.

[13] S. Wicker, Error Control Systems for Digital Communication and Storage, Prentice Hall, 1995.

[14] J. Janssen, D. De Vleeschauwer, M. Bchli, and G. H. Petit, "Assessing voice quality in packet-based telephony," IEEE Internet Computing, pp. 48-56, May-June 2002.

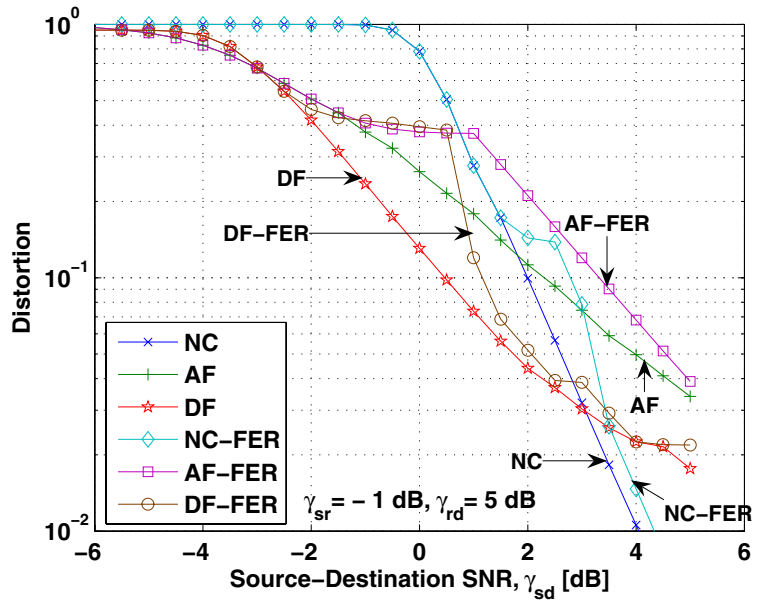

(a) $\gamma_{s r}=-1 d B, \gamma_{r d}=5 d B$

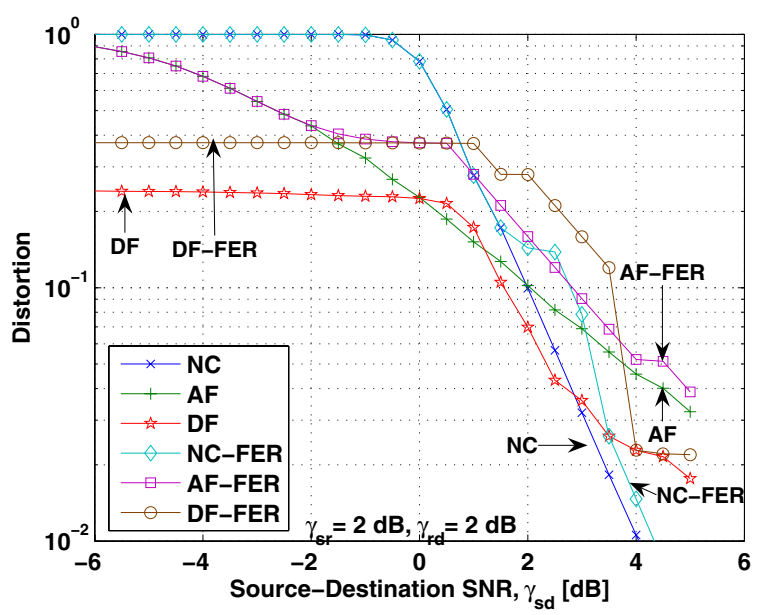

(b) $\gamma_{s r}=\gamma_{r d}=2 d B$

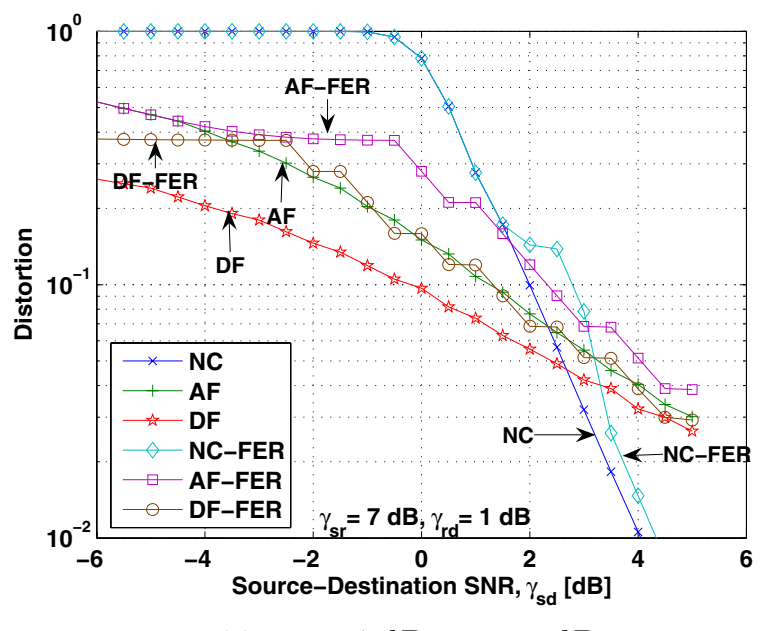

(c) $\gamma_{s r}=7 d B, \gamma_{r d}=1 d B$

Fig. 6. D-SNR curves with and without QoS constraint with FER $Q=10^{-3}$. 